\title{
Is a Positive Family History of Endometriosis a Risk Factor for Endometrioma Recurrence After Laparoscopic Surgery?
}

Reproductive Sciences

2014, Vol. 2I(4) 526-53।

(C) The Author(s) 2013

Reprints and permission:

sagepub.com/journalsPermissions.nav DOI: $10.1177 / 1933719113503413$

rs.sagepub.com

(S)SAGE

\author{
Sebastiano Campo, MD', Vincenzo Campo, MD, PhD', \\ and Pietro Gambadauro, MD, PhD ${ }^{2}$
}

\begin{abstract}
A total of 148 patients were followed up for an average of $30.1 \pm 17$ months following to laparoscopic excision of ovarian endometriomas by a single surgical team. Bivariate and multivariate analyses were used to investigate the association between endometrioma recurrence and several factors, age, body mass index, family history, cyst diameter, number and location, adhesions or peritoneal implants, occurrence of spillage, postoperative treatment with gonadotropin-releasing hormone agonist, or pregnancies. The overall recurrence rate of the endometriomas was $18.2 \%$. At bivariate analysis, recurrence rate was significantly higher in patients with a positive family history of endometriosis (40\% vs $14.8 \%$ ). Recurrence was also more frequent, albeit nonsignificantly, in patients with a history of dysmenorrhea, intraoperative spillage, and postoperative hormonal suppression. At multivariate analysis with logistic regression, a positive family history of endometriosis was the only variable independently associated with endometrioma recurrence following laparoscopic removal (odds ratio 3.245; 95\% confidence interval: I.090-9.66I).
\end{abstract}

\section{Keywords}

endometrioma, endometriosis, laparoscopy, recurrence, family history

\section{Introduction}

Endometriosis currently represents one of the major problems faced by gynecologists and reproductive endocrinologists. Its prevalence is estimated at $2 \%$ to $22 \%$ among asymptomatic women, $40 \%$ to $60 \%$ in women with dysmenorrhoea or chronic pelvic pain, and $20 \%$ to $30 \%$ in infertile women. ${ }^{1}$ In affected patients, ovarian endometriomas, or chocolate cysts, are common. To date, no definitive cure for endometriosis is available, thus the aims of treatment are to decrease patients' pain, to enhance their fertility, and ideally, to delay the recurrence of disease. Conservative medical treatment leads to a reduction in volume rather than a complete regression of endometriotic cysts. ${ }^{2}$ This is probably caused by the persistence of endometriotic tissue notwithstanding the medical treatment. ${ }^{2}$ For this reason, surgery is the most effective therapeutic strategy, ${ }^{3}$ and, in particular, laparoscopy represents the gold standard for the treatment of ovarian endometriotic cysts. ${ }^{4,5}$

The aim of this study was to evaluate the factors associated with recurrence after laparoscopic excision of ovarian endometriomas.

\section{Materials and Methods}

From January 2001 to January 2006, 265 patients with a clinical and/or ultrasonographic diagnosis of endometriosis underwent laparoscopy under our care at the Department of Obstetrics and Gynecology of the Catholic University of the Sacred Heart,
Rome, Italy. Of this population, a cohort of 149 patients with ovarian endometriomas removed at surgery was identified to perform this study. Only patients with at least 1 ovarian endometrioma $\geq 2 \mathrm{~cm}$ in diameter were included, while patients with the following characteristics were excluded from the study: minimal or mild endometriosis, rectovaginal endometriosis, neoadjuvant treatment with estroprogestins or gonadotropin-releasing hormone (GnRH) analogs, follow-up period under 12 months. In all cases, the preoperative diagnosis was based on the clinical parameters and on the findings of transvaginal ultrasound by experienced gynecologists. All the endometriomas were confirmed by the pathology report.

Laparoscopy was performed with the patient under general anesthesia, using a 10-mm laparoscope (Karl Storz $\mathrm{GmbH}$ and Co, Tuttlingen, Germany) that was introduced into the peritoneal cavity through the umbilicus and connected to a videocamera. Three 5-mm trocars were used for ancillary instruments. An endouterine mobilizer was applied when it was possible. The

\footnotetext{
I Institute of Obstetrics and Gynecology, Catholic University of the Sacred Heart, Rome, Italy

${ }^{2}$ Centre for Reproduction, Uppsala University Hospital, Uppsala, Sweden

Corresponding Author:

Pietro Gambadauro, Luthagsesplanaden 24b, 75224 Uppsala, Sweden.

Email: gambadauro@gmail.com
} 
Table I. Characteristics of the Study Population.

\begin{tabular}{|c|c|c|}
\hline Number of patients ${ }^{\mathrm{a}}$ & 148 & $100 \%$ \\
\hline Age, ${ }^{a}$ years & $32.07 \pm 6.9$ & $95 \% \mathrm{Cl}: 30.95-33.19$ \\
\hline $\mathrm{BMI}^{\mathrm{a}}$ & $21.79 \pm 2.7$ & $95 \%$ Cl: $21.3-22.2$ \\
\hline Number of endometriomas ${ }^{a}$ & $1.37 \pm 0.64$ & $95 \% \mathrm{Cl}: 1.27-1.48$ \\
\hline $\operatorname{Size}^{\mathrm{a}}(\mathrm{cm})$ & $4.88 \pm 1.9$ & $95 \% \mathrm{Cl}: 4.57-5.19$ \\
\hline \multicolumn{3}{|l|}{ Location ${ }^{\mathrm{b}}$} \\
\hline Right & 44 & $29.7 \%$ \\
\hline Left & 73 & $49.3 \%$ \\
\hline Bilateral & 31 & $20.9 \%$ \\
\hline Adhesions $^{b}$ & 100 & $67.6 \%$ \\
\hline Peritoneal implants ${ }^{\mathrm{b}}$ & 62 & $41.9 \%$ \\
\hline Operative time, ${ }^{\mathrm{a}} \mathrm{min}$ & $75.14 \pm 31.4$ & $95 \% \mathrm{Cl}: 70.03-80.24$ \\
\hline Postoperative stay, ${ }^{a}$ days & $1.28 \pm 0.629$ & $95 \%$ Cl: I.I8-I.39 \\
\hline Complications $^{\mathrm{b}}$ & $\overline{2}$ & $1.35 \%$ \\
\hline Follow-up, ${ }^{a}$ months & $30.1 \pm 17.0$ & $95 \% \mathrm{Cl}: 27.33-32.87$ \\
\hline Recurrence $^{\mathrm{b}}$ & $\overline{27}$ & $18.2 \%$ \\
\hline
\end{tabular}

Abbreviation: BMI, body mass index.

a Mean \pm standard deviation.

b Number of cases.

pelvis, the abdomen, and the external surface of the cysts were systematically examined to exclude signs of malignancy. In all patients, the cysts were removed using the stripping technique. ${ }^{6}$ After identifying the cleavage plane, the endometrial cyst was opened, and the cystic content was immediately aspirated taking care to minimize spillage. Series of suctions and washings were performed with a suction-irrigation cannula introduced into the cyst. Washings were continued until the rinsing solution became clear. The cyst capsule was separated from the ovarian cortex by means of diverging tractions applied using 2 atraumatic forceps. Bipolar coagulation was used for hemostasis only when necessary. The cyst capsule was removed from the abdominal cavity using a disposable endoscopic bag and sent for histological analysis. No sutures were used to approximate the margins of the ovarian defect. During surgery, if necessary, adhesiolysis and coagulation of peritoneal implants were performed with bipolar forceps. Infertile patients were also submitted to diagnostic hysteroscopy as well as to bilateral chromotubation to check the tubal patency at the end of the laparoscopic procedure.

The follow-up period included clinical and sonographic evaluation performed by a specialist gynecologist twice yearly for the first 2 years after the intervention and then on a yearly basis unless the cyst recurred. This was an observational study, and all the interventions described previously were part of the routine care of patients with endometriosis in our group. All patients gave their informed consent before surgery.

For all the patients, epidemiological, anamnestic, and clinical data were collected and stored on a dedicated database. Age (years) and body mass index (BMI; $\mathrm{kg} / \mathrm{m}^{2}$ ) were treated as continuous variables. Dysmenorrhea, infertility, previous pregnancies and parity, and family history of endometriosis (up to third-degree relatives) were considered as categorical variables (yes/no). We also retrieved data on the endometriomas and the surgical procedures, such as cyst diameter $(\mathrm{cm})$, number of cysts per patient, presence and treatment of adhesions or peritoneal implants, operative time (minutes), intraoperative spillage of cystic contents, and postoperative hospital stay (days). The following follow-up data were considered: use of hormonal suppression treatments, pregnancies and parity, recurrence of endometriomas, and time to the last follow-up visit. Recurrence of endometrioma was defined as the detection, at transvaginal ultrasound, of a persistent cyst with the typical sonographic features of endometrioma measuring $\geq 2$ $\mathrm{cm}$ in diameter, within the ovarian parenchyma.

Data were initially analyzed by descriptive statistics. We have calculated frequencies and percentages for categorical variables and mean, standard deviation (SD), and 95\% confidence intervals (CIs) for continuous variables. A bivariate analysis was performed to evaluate the differences in recurrence for continuous and categorical variables. Mann-Whitney $U$ test was used for continuous variables, while Fisher exact and chi-square tests were used for categorical variables, as appropriate. Logistic regression was used to study the independent association between recurrence of endometriomas and the variables, which resulted as associated at bivariate analysis $(P<$ .1). Differences were considered statistically significant in case of a $P$ value $<.05$ (2-tailed). Odds ratio (OR) was used to express the strength of associations, together with $95 \%$ CI.

The statistical analyses were performed on the software SPSS Statistics v20 (IBM) for Mac OSX, and manually.

\section{Results}

The mean age of the 148 patients included in the study was $32.07 \pm 6.9$ years $(95 \%$ CI 30.95-33.19). General details of the study group are presented in Table 1. Overall, 203 endometriomas were excised. The mean number of endometrioma per patient was $1.37 \pm 0.64$ (range 1-4). The cysts were located only on the right ovary in the $29.7 \%$ of cases and only on the left ovary in the $49.3 \%$. Bilateral endometriomas were present in the $20.9 \%$ of the patients. The mean diameter of the cysts was $4.88 \pm 1.9 \mathrm{~cm}$. Pelvic or adnexal adhesions were found in the $67.6 \%$ (100 of 148) of the patients. Sixty two (41.9\%) patients had peritoneal implants of endometriosis.

The mean operative time was $75.14 \pm 31.4$ minutes. The mean postoperative hospital stay was $1.28 \pm 0.629$ days. Two $(1.35 \%)$ patients developed postoperative complications, 1 patient had severe anemia and the other, who had undergone extensive adhesiolysis, presented with intestinal occlusion 20 days after the surgical procedure. Forty-six women received postoperative hormonal suppression by GnRH analogs during 6 months, according to the preoperative recommendation of their referring physician. This decision was not influenced by intraoperative findings.

All the patients were followed up for at least 12 months after the laparoscopic surgery. The mean length of the follow-up was 30.1 months ( \pm 17.0 SD; $95 \%$ CI: 27.33-32.87). Recurrence of ovarian endometrioma was detected in $27(18.2 \%)$ patients at transvaginal ultrasound.

At bivariate analysis with Mann-Whitney $U$ test, no significant differences were found between patients with and without 
Table 2. Comparison Between Patients With and Without Endometrioma Recurrence.

\begin{tabular}{|c|c|c|c|}
\hline \multirow[b]{2}{*}{ Variables } & \multicolumn{2}{|c|}{ Recurrence } & \multirow[b]{2}{*}{$P$} \\
\hline & Yes (27) & No $(12 I)$ & \\
\hline Age, mean $\pm S D$, years & $30.8 \pm 5.6$ & $32.3 \pm 7.2$ & $.376^{\mathrm{a}}$ \\
\hline $\mathrm{BMI}$, mean $\pm \mathrm{SD}$ & $21.5 \pm 2.4$ & $21.8 \pm 2.7$ & $.520^{\mathrm{a}}$ \\
\hline Number of cysts, mean $\pm S D$ & $1.44 \pm 0.6$ & $1.36 \pm 0.6$ & $.375^{\mathrm{a}}$ \\
\hline Max diameter, mean $\pm \overline{\mathrm{SD}}, \mathrm{cm}$ & $5.39 \pm 2.1$ & $4.76 \pm 1.8$ & $.159^{\mathrm{a}}$ \\
\hline \multicolumn{4}{|c|}{ Dysmenorrhea } \\
\hline Yes & 22 & 75 & $.072^{\mathrm{b}}$ \\
\hline No & 5 & 46 & \\
\hline \multicolumn{4}{|l|}{ Infertility } \\
\hline Yes & 5 & 30 & $.62^{\mathrm{b}}$ \\
\hline No & 22 & 91 & \\
\hline \multicolumn{4}{|l|}{ Preoperative parity } \\
\hline Yes & 6 & 36 & $.433^{\mathrm{c}}$ \\
\hline No & 21 & 85 & \\
\hline \multicolumn{4}{|l|}{ Positive family history } \\
\hline Yes & 8 & 12 & $.007^{\mathrm{c}}$ \\
\hline No & 19 & 109 & \\
\hline \multicolumn{4}{|l|}{ Cyst location } \\
\hline Right & 5 & 39 & $.359^{c}$ \\
\hline Left & 15 & 58 & \\
\hline Bilateral & 7 & 24 & \\
\hline \multicolumn{4}{|l|}{ Adhesions } \\
\hline Yes & 19 & 81 & $.731^{c}$ \\
\hline No & 8 & 40 & \\
\hline \multicolumn{4}{|l|}{ Peritoneal implants } \\
\hline Yes & 10 & 52 & $.572^{\mathrm{c}}$ \\
\hline No & 17 & 69 & \\
\hline \multicolumn{4}{|l|}{ Spillage } \\
\hline Yes & 17 & 51 & $.050^{c}$ \\
\hline No & 10 & 70 & \\
\hline \multicolumn{4}{|l|}{ Postoperative treatment } \\
\hline Yes & 12 & 34 & $.097^{\mathrm{c}}$ \\
\hline No & 15 & 87 & \\
\hline \multicolumn{4}{|l|}{ Postoperative pregnancy } \\
\hline Yes & 4 & 25 & $.599^{\mathrm{b}}$ \\
\hline No & 23 & 96 & \\
\hline \multicolumn{4}{|l|}{ Postoperative parity } \\
\hline Yes & 3 & 22 & $.570^{\mathrm{b}}$ \\
\hline No & 24 & 99 & \\
\hline
\end{tabular}

Abbreviations: BMI, body mass index; SD, standard deviation.

${ }^{\text {a }}$ Mann-Whitney $U$ test.

${ }^{b}$ Fisher exact test.

c Chi-square test.

recurrence in terms of age, BMI, cyst diameter, and number of endometriomas per patient (Table 2).

Recurrence of endometrioma was significantly associated with a positive familial anamnesis for endometriosis. Recurrence rate was $40 \%$ in patients with a positive family history versus $14.8 \%$ in patients with no familial anamnesis for endometriosis ( $P .007$; Table 2). Higher recurrence rates were also found in patients with a history of dysmenorrhea $(22.7 \%$ vs $9.8 \%)$, intraoperative spillage ( $25 \%$ vs $12.5 \%$ ), and postoperative hormonal suppression (26\% vs $14.7 \%$ ), although those differences were not statistically significant $(P \geq .05$; Table 2$)$. A longer
Table 3. Factors Associated With Endometrioma Recurrence Following Laparoscopic Surgery.

\begin{tabular}{lccc}
\hline & OR & $95 \% \mathrm{Cl}$ & $P$ \\
\hline Dysmenorrhea & $2.50 \mathrm{I}$ & $0.850-7.357$ & .096 \\
Positive family history & 3.245 & $1.090-9.66 \mathrm{I}$ & .035 \\
Spillage & 1.557 & $0.588-4.120$ & .373 \\
Postoperative treatment & 1.372 & $0.504-3.736$ & .536 \\
Length of follow-up & 1.022 & $0.995-1.05 \mathrm{I}$ & .112 \\
\hline
\end{tabular}

Abbreviations: $\mathrm{Cl}$, confidence interval; OR, odds ratio.

Table 4. Comparison Between Patients With and Without a Positive Family History of Endometriosis.

\begin{tabular}{lccc}
\hline & \multicolumn{2}{c}{ Family History } \\
\cline { 2 - 3 } & Positive & Negative & \\
\cline { 2 - 3 } Number of cases & 20 & 128 & \\
Age, years & $31.95 \pm 7.85$ & $32.09 \pm 6.77$ & $.755^{\mathrm{a}}$ \\
BMI & $22.19 \pm 2.36$ & $21.73 \pm 2.76$ & $.356^{\mathrm{a}}$ \\
Number of cysts & $1.55 \pm 0.82$ & $1.34 \pm 0.60$ & $.254^{\mathrm{a}}$ \\
Max diameter, cm & $5.02 \pm 2.55$ & $4.86 \pm 1.79$ & $.788^{\mathrm{a}}$ \\
Follow-up, months & $32.85 \pm 17.90$ & $29.67 \pm 16.96$ & $.443^{\mathrm{a}}$ \\
Dysmenorrhea & $16(80 \%)$ & $81(63.2 \%)$ & $.206^{\mathrm{b}}$ \\
Infertility & $5(25 \%)$ & $30(23.4 \%)$ & $>.99^{\mathrm{b}}$ \\
Preoperative parity & $5(25 \%)$ & $37(28.9 \%)$ & $.796^{\mathrm{b}}$ \\
Cyst location & & & \\
$\quad$ Right & $6(30 \%)$ & $38(29.7 \%)$ & $.994^{\mathrm{c}}$ \\
$\quad$ Left & $10(50 \%)$ & $63(49.2 \%)$ & \\
$\quad$ Bilateral & $4(20 \%)$ & $27(21.1 \%)$ & \\
Adhesions & $15(75 \%)$ & $85(66.4 \%)$ & $.609^{\mathrm{b}}$ \\
Peritoneal implants & $7(35 \%)$ & $55(42.9 \%)$ & $.502^{\mathrm{c}}$ \\
Spillage & $11(55 \%)$ & $57(44.5 \%)$ & $.382^{\mathrm{c}}$ \\
Postoperative treatment & $6(30 \%)$ & $40(31.2 \%)$ & $.911^{\mathrm{c}}$ \\
Postoperative pregnancy & $5(25 \%)$ & $24(18.7 \%)$ & $.547^{\mathrm{b}}$ \\
Postoperative parity & $5(25 \%)$ & $20(15.6 \%)$ & $.336^{\mathrm{b}}$ \\
Recurrence & $8(40 \%)$ & $19(14.8 \%)$ & $.007^{\mathrm{c}}$ \\
\hline Abbrevary & & &
\end{tabular}

Abbreviation: BMI, body mass index.

a Mann-Whitney $U$ test.

${ }^{\mathrm{b}}$ Fisher exact test.

${ }^{c}$ Chi-square test.

follow-up was associated with cyst recurrence. No differences in recurrence rate were found when assessing infertility anamnesis, cyst location, adhesions or peritoneal implants, postoperative pregnancies, and parity.

At multivariate analysis with logistic regression, the only variable showing a statistically significant association with the recurrence of endometrioma was the positive family history of endometriosis (OR 3.245; 95\% CI: 1.090-9.661; P .035; Table 3). No significant differences were found between patients with and without a positive family history, except for recurrence rate (Table 4).

\section{Discussion}

The laparoscopic removal of ovarian endometriomas by stripping of the cyst capsule is an established technique. ${ }^{7}$ In the present series, we operated laparoscopically 148 women with 
endometriomas, and we report operative times similar to what previously published, ${ }^{4}$ short time to patient discharge, and low complication rate $(1.35 \%)$.

In spite of the technical safety and efficacy of this type of surgery, recurrence of the endometrioma is not uncommon, and it is obviously frustrating for the patients and their surgeons. This might be related to the characteristics of the disease itself rather than to surgery, and recurrence rates following conservative surgery for ovarian endometriomas have been reported to range between $8 \%$ and $40 \%{ }^{8}$ In our experience including 148 patients, the recurrence rate at a mean of 30-month follow-up was $18.2 \%$. Recurrence rate is obviously affected by the definition of endometrioma that is used, and heterogeneity in this subject is present in the published literature. The recurrence rate in our series, where endometriomas $\geq 2 \mathrm{~cm}$ were considered, was similar to what previously reported by Alborzi et $\mathrm{al}^{5}$ and Ghezzi et al ${ }^{9}$ who defined recurrence as a persistent cyst $>3 \mathrm{~cm}$ or $\geq 4 \mathrm{~cm}$ in diameter, respectively. Interestingly, both the groups reported an overall recurrence rate of $17.3 \%$, respectively, after 2 and 3 years of follow-up. On the contrary, other authors have reported recurrence rates up to $26 \%$ after a follow-up of less than 2 years, but when a diameter of $1 \mathrm{~cm}$ was considered as a lower limit for cyst recurrence. ${ }^{10}$

Previous reports have attempted to identify risk or protective factors for endometrioma recurrence. Pregnancy after surgery has been identified as a possible protective factor by various authors. ${ }^{10-15}$ Studies on the relationship between hormonal suppression by $\mathrm{GnRH}$ analogs and endometrioma recurrence have given controversial results. The preoperative use of GnRH ana$\operatorname{logs}$ has been associated with higher recurrence rate. ${ }^{11}$ Some authors have found a similar increase in endometrioma recurrence rate also with postoperative treatment. ${ }^{10,16}$ Others have instead demonstrated no benefit of 6 months of hormonal suppression over placebo in terms of recurrence. ${ }^{17,18}$ On the contrary, GnRH agonists seem to delay the occurrence of disease recurrence after surgery. ${ }^{17}$ A protective effect of postoperative contraceptives has been reported. ${ }^{19-21}$

A study reported a 3 -fold higher risk of recurrence in women older than 30 years as compared to those aged 20 to 30 years, ${ }^{22}$ although this has not been confirmed by others. ${ }^{9-11}$ In fact, a recent study by Sengoku et al has shown younger age at surgery as a risk factor for recurrence. ${ }^{14}$

A higher recurrence rate of endometriomas has been seen in case of left-side or bilateral cysts, ${ }^{9,23}$ and when adnexal or culde-sac adhesions are present. ${ }^{10}$

The variety of suggested risk/protective factors, and the heterogeneity of the published results, certainly depends on the characteristics of endometriosis as a disease. Both eutopic and ectopic endometria of women with endometriosis are variably different from the endometrium of healthy women and might also therefore respond in unusual and heterogeneous ways to physiological hormonal changes. ${ }^{24}$ Great clinical variability exists between patients, and a certain grade of unpredictability is linked to endometriosis. ${ }^{25}$ In some women, adenomyosis, a still poorly understood and possibly underdiagnosed condition, might complicate the picture and be responsible for the disappointing persistence of symptoms after appropriate or even radical, uterus-sparing surgery. ${ }^{26-28}$

In this study, we have tried to focus on several possible factors but have failed to demonstrate a significant association of endometrioma recurrence with most of the studied variables. We cannot confirm the role of postoperative pregnancy as a protective factor. Our patients had not received hormonal pretreatment, and no differences in recurrence rates were found between patients who where treated postoperatively and those who were not. We did not find significant difference in age, BMI, size, number, or location of the cyst. Moreover, we could not see any statistical effect of adhesions and peritoneal implants on the risk of the recurrence, although we saw an increased, albeit nonsignificantly, risk in patients where a frank spillage of cyst contents occurred during surgery.

Nevertheless, we have observed a statistically significant association between a positive family history of endometriosis and endometrioma recurrence. We believe that this is an interesting and, to the best of our knowledge, novel finding, supporting the hypothesis of a genetic basis both to endometriosis and to its clinical behavior.

An early survey-based study showing a hereditary tendency in endometriosis was published already in 1971 by Ranney. ${ }^{29}$ Since then, various reports on this subject have been published. Clinical studies support the hypothesis of heritability of endometriosis. A 4- to 10-fold increased risk of endometriosis has been demonstrated in first-degree relatives of patients, ${ }^{30-33}$ and endometriosis is more common among monozygotic twins compared to dizygotic twins. ${ }^{34}$ On the other hand, genome-wide association studies have started to identify loci for endometriosis. ${ }^{35,36}$ Interestingly, not only the occurrence but also the severity of endometriosis has been related to heritability. Moen and Magnus reported already 20 years ago how an aggressive behavior of endometriosis, in terms of clinical manifestations, was significantly more common among patients with a positive family history. ${ }^{33}$ This is similar to what we found in this study, where the recurrence rates in patient with and without a positive family history were, respectively, $40 \%$ and $14.8 \%$.

To the best of our knowledge, our study is the first report of an association between family history and recurrence rate of endometrioma following surgery. This is pointing toward a higher grade of severity of endometriosis in familial cases, and in our opinion, deserves further studies. A limit of our experience is obviously represented by its retrospective nature, which exposes our results to the risk of bias. Nevertheless, all the laparoscopies were performed by the same team, thus tackling performance bias. Moreover, we have minimized the risk of confounders by performing a multivariate analysis, where various factors were assessed, including follow-up time. Our logistic regression model has confirmed the independent association of a positive family history of endometriosis with endometrioma recurrence.

In conclusion, our results show that laparoscopic removal of endometriomas is safe but carries a risk of recurrence. A positive family history of endometriosis is independently associated with the risk of recurrence of endometrioma following 
laparoscopic surgery. Further prospective studies are needed to confirm these results.

\section{Authors' Note}

This study was performed at Institute of Obstetrics and Gynecology, Catholic University of the Sacred Heart, Rome, Italy.

\section{Declaration of Conflicting Interests}

The author(s) declared no potential conflicts of interest with respect to the research, authorship, and/or publication of this article.

\section{Funding}

The author(s) received no financial support for the research, authorship, and/or publication of this article.

\section{References}

1. Farquhar CM. Extracts from the "clinical evidence". Endometriosis. BMJ. 2000;320(7247):1449-1452.

2. Donnez J, Nisolle-Pochet M, Clerckx-Braun F, Sandow J, Casanas-Roux F. Administration of nasal buserelin as compared with subcutaneous buserelin implant for endometriosis. Fertil Steril. 1989;52(1):27-30.

3. Farquhar C, Sutton C. The evidence for the management of endometriosis. Curr Opin Obstet Gynecol. 1998;10(4):321-332.

4. Beretta P, Franchi M, Ghezzi F, Busacca M, Zupi E, Bolis P. Randomized clinical trial of two laparoscopic treatments of endometriomas: cystectomy versus drainage and coagulation. Fertil Steril. 1998;70(6):1176-1180.

5. Alborzi S, Momtahan M, Parsanezhad ME, Dehbashi S, Zolghadri J, Alborzi S. A prospective, randomized study comparing laparoscopic ovarian cystectomy versus fenestration and coagulation in patients with endometriomas. Fertil Steril. 2004;82(6):1633-1637.

6. Canis M, Mage G, Wattiez A, Chapron C, Pouly JL, Bassil S. Second-look laparoscopy after laparoscopic cystectomy of large ovarian endometriomas. Fertil Steril. 1992;58(3):617-619.

7. Hart RJ, Hickey M, Maouris P, Buckett W. Excisional surgery versus ablative surgery for ovarian endometriomata. Cochrane Database Syst Rev. 2008;(2):CD004992.

8. American College of Obstetrician and Gynecologist. Endometriosis. Endometriosis. ACOG technical bulletin number 184-September 1993. Int J Gynecol Obstet. 1993;43(2):221-227.

9. Ghezzi F, Beretta P, Franchi M, Parissis M, Bolis P. Recurrence of ovarian endometriosis and anatomical location of the primary lesion. Fertil Steril. 2001;75(1):136-140.

10. Porpora MG, Pallante D, Ferro A, Crisafi B, Bellati F, Benedetti Panici P. Pain and ovarian endometrioma recurrence after laparoscopic treatment of endometriosis: a long-term prospective study. Fertil Steril. 2010;93(3):716-721.

11. Koga K, Takemura Y, Osuga Y, et al. Recurrence of ovarian endometrioma after laparoscopic excision. Hum Reprod. 2006; 21(8):2171-2174.

12. Busacca M, Chiaffarino F, Candiani M, et al. Determinants of longterm clinically detected recurrence rates of deep, ovarian, and pelvic endometriosis. Am J Obstet Gynecol. 2006;195(2):426-432.

13. Coccia ME, Rizzello F, Palagiano A, Scarselli G. Long-term followup after laparoscopic treatment for endometriosis: multivariate analysis of predictive factors for recurrence of endometriotic lesions and pain. Eur J Obstet Gynecol Reprod Biol. 2011;157(1):78-83.

14. Sengoku K, Miyamoto T, Horikawa M, et al. Clinicopathologic risk factors for recurrence of ovarian endometrioma following laparoscopic cystectomy. Acta Obstet Gynecol Scand. 2013;92(3): 278-284.

15. Hayasaka S, Ugajin T, Fujii O, et al. Risk factors for recurrence and re-recurrence of ovarian endometriomas after laparoscopic excision. J Obstet Gynaecol Res. 2011;37(6):581-585.

16. Loverro G, Carriero C, Rossi AC, Putignano G, Nicolardi V, Selvaggi L. A randomized study comparing triptorelin or expectant management following conservative laparoscopic surgery for symptomatic stage III-IV endometriosis. Eur J Obstet Gynecol Reprod Biol. 2008;136(2):194-198.

17. Jee BC, Lee JY, Suh CS, Kim SH, Choi YM, Moon SY. Impact of GnRH agonist treatment on recurrence of ovarian endometriomas after conservative laparoscopic surgery. Fertil Steril. 2009;91(1): 40-45.

18. Sesti F, Capozzolo T, Pietropolli A, Marziali M, Bollea MR, Piccione E. Recurrence rate of endometrioma after laparoscopic cystectomy: a comparative randomized trial between postoperative hormonal suppression treatment or dietary therapy vs. placebo. Eur J Obstet Gynecol Reprod Biol. 2009;147(1):72-77.

19. Vercellini P, Somigliana E, Daguati R, Vigano P, Meroni F, Crosignani PG. Postoperative oral contraceptive exposure and risk of endometrioma recurrence. Am J Obstet Gynecol. 2008;198(5): 504.e1-504.e5.

20. Seracchioli R, Mabrouk M, Frascà C, et al. Long-term cyclic and continuous oral contraceptive therapy and endometrioma recurrence: a randomized controlled trial. Fertil Steril. 2010;93(1): $52-56$.

21. Takamura M, Koga K, Osuga Y, et al. Post-operative oral contraceptive use reduces the risk of ovarian endometrioma recurrence after laparoscopic excision. Hum Reprod. 2009;24(12):3042-3048.

22. Parazzini F, Bertulessi C, Pasini A, et al. Determinants of short term recurrence rate of endometriosis. Eur J Obstet Gynecol Reprod Biol. 2005;121(2):216-219.

23. Jones KD, Sutton CJ. Recurrence of chocolate cysts after laparoscopic ablation. J Am Assoc Gynecol Laparosc. 2002;9(3): 315-320.

24. Brosens I, Brosens JJ, Benagiano G. The eutopic endometrium in endometriosis: are the changes of clinical significance? Reprod Biomed Online. 2012;24(5):496-502.

25. Gambadauro P, Olovsson M, Persson P. Unusually rapid growth of bilateral endometriomas and acute bilateral hydronephrosis. Gynecol Endocrinol. 2011;27(11):948-950.

26. Brosens I, Kunz G, Benagiano G. Is adenomyosis the neglected phenotype of an endo-myometrial dysfunction syndrome? Gynecol Surg. 2012;9(2):131-137.

27. Parker JD, Leondires M, Sinaii N, Premkumar A, Nieman LK, Stratton P. Persistence of dysmenorrhea and nonmenstrual pain after optimal endometriosis surgery may indicate adenomyosis. Fertil Steril. 2006;86(3):711-715.

28. Brosens I, Benagiano G. Poor results after surgery for rectovaginal endometriosis can be related to uterine adenomyosis. Hum Reprod. 2012;27(11):3360-3361. 
29. Ranney B. Endometriosis. IV. Hereditary tendency. Obstet Gynecol. 1971;37(5):734-737.

30. Matalliotakis IM, Arici A, Cakmak H, Goumenou AG, Koumantakis G, Mahutte NG. Familial aggregation of endometriosis in the Yale Series. Arch Gynecol Obstet. 2008; 278(6):507-511.

31. Simpson JL, Elias S, Malinak LR, Buttram VC. Heritable aspects of endometriosis. 1. Genetic studies. Am J Obstet Gynecol. 1980;137(3):327-331.

32. Lamb RN, Hoffmann RG, Nichols TR. Family trait analysis: a case-control study of 43 women with endometriosis and their best friends. Am J Obstet Gynecol. 1986;154(3):596-601.
33. Moen MH, Magnus P. The familial risk of endometriosis. Acta Obstet Gynecol Scand. 1993;72(7):560-564.

34. Treloar SA, O'Connor DT, O'Connor VM, Martin NG. Genetic influences on endometriosis in an Australian twin sample. Fertil Steril. 1999;71(4):701-710.

35. Pagliardini L, Gentilini D, Vigano' $P$, et al. An Italian association study and meta-analysis with previous GWAS confirm WNT4, CDKN2BAS and FN1 as the first identified susceptibility loci for endometriosis. J Med Genet. 2013;50(1):43-46.

36. Nyholt DR, Low SK, Anderson CA, et al. Genome-wide association meta-analysis identifies new endometriosis risk loci. Nat Genet. 2012;44(12):1355-1359. 